Radiologe 2010 · 50:1120-1127

DOI 10.1007/s00117-010-2053-2

Online publiziert: 17. September 2010

๑) Springer-Verlag 2010

S.T. Schindera ${ }^{1}$ C. Nauer ${ }^{2}$ R. Treier ${ }^{3} \cdot$ P. Trueb ${ }^{3} \cdot$ G. von Allmen ${ }^{1} \cdot$ P. Vock ${ }^{1}$.

Z. Szucs-Farkas ${ }^{1}$

${ }^{1}$ Institut für Diagnostische, Interventionelle und Pädiatrische Radiologie,

Universitätsspital Bern, Inselspital, Bern

${ }^{2}$ Institut für Diagnostische und Interventionelle Neuroradiologie,

Universitätsspital Bern, Inselspital, Bern

${ }^{3}$ Abteilung Strahlenschutz, Bundesamt für Gesundheit, Bern

\title{
Strategien zur Reduktion der CT-Strahlendosis
}

der erforderlichen Fachkunde im Strahlenschutz gestellt werden. Zudem sollten immer diagnostische Alternativen ohne Strahlenexposition (Z. B. Ultraschall, $M R T$ ) in Erwägung gezogen werden. Für Radiologen und ihre klinischen Zuweiser gibt es verschiedene Orientierungshilfen. Diese sollen helfen, diejenigen bildgebenden Verfahren auszuwählen, die für die jeweilige Fragestellung am besten geeignet sind [3, 4]. Das Befolgen der Orientierungshilfe lässt neben einer besseren klinischen Versorgung der Patienten auch eine Verringerung der damit verbundenen Strahlenexposition erwarten.

\section{Einschränkung der Scanlänge}

Die Scanregion soll ausschließlich denjenigen Körperbereich abdecken, der für die Beantwortung der klinischen Fragestellung notwendig ist (• Abb. 1). Im klinischen Alltag kommt es häufig vor, dass die Scanlänge zu groß definiert und damit eine zusätzliche, unnötige Strahlenexposition verursacht wird. Oftmals spielt hier die Befürchtung eine Rolle, wichtige anatomische Strukturen oder auch Pathologien zu verpassen. Eine Instruktion der MTRA bzgl. einer exakten Planung des Scanbereichs abhängig von der klinischen Fragestellung ist wichtig und trägt zur Reduktion der Strahlenbelastung bei. Die Begrenzung der Scanlänge hat direkten Einfluss auf das Dosislängenprodukt (Scanlänge $\times$ gewichteter CT-Dosisindex). Das Dosislängenprodukt ist ein Maß für die effektive Patientendosis und wird für jede
CT-Untersuchung auf der CT-Scannerkonsole angegeben.

Kalra et al. [5] konnten zeigen, dass in bis zu 97\% der Fälle einer CT-Untersuchung des Abdomens und Beckens der Scanbereich zu großzügig gewählt wurde. Bei der CT-Untersuchung des Abdomens (insbesondere bei weiblichen Patienten) sollte wegen des strahlensensitiven Brustdrüsengewebes die Körperregion oberhalb des Diaphragmas nur knapp miterfasst werden. Bei der CT-Untersuchung des Beckens (insbesondere beim männlichen Patienten) sollten die strahlensensitiven Gonaden nicht routinemäßig dargestellt werden. Bei der CT-Untersuchung des Halses sollte das Viszerokranium inklusive der Augenlinsen nur miterfasst werden, wenn es für die klinische Fragestellung notwendig ist.

Bei der Lungenemboliediagnostik kann in Einzelfällen (Z. B. schwangere Patientinnen, sehr junge Patienten) die Scanlänge deutlich reduziert werden, indem der Lungenapex und die Lungenbasis nicht in die Untersuchung einbezogen werden (• Abb. 2). Eine Lungenembolie in diesen peripheren Regionen betrifft nur kleine subsegmentale Lungenarterien und hat keinen Einfluss auf die Therapie. Durch die Einschränkung der Scanlänge bei der Lungenemboliediagnostik kann eine Dosisreduktion bis zu 30\% erreicht werden [6]. Jedoch steigt durch die Einschränkung der Scanlänge das Risiko, eine eventuelle Raumforderung im Lungenapex oder in der Lungenbasis nicht zu entdecken. 
Hier steht eine Anzeige.

黛 Springer 


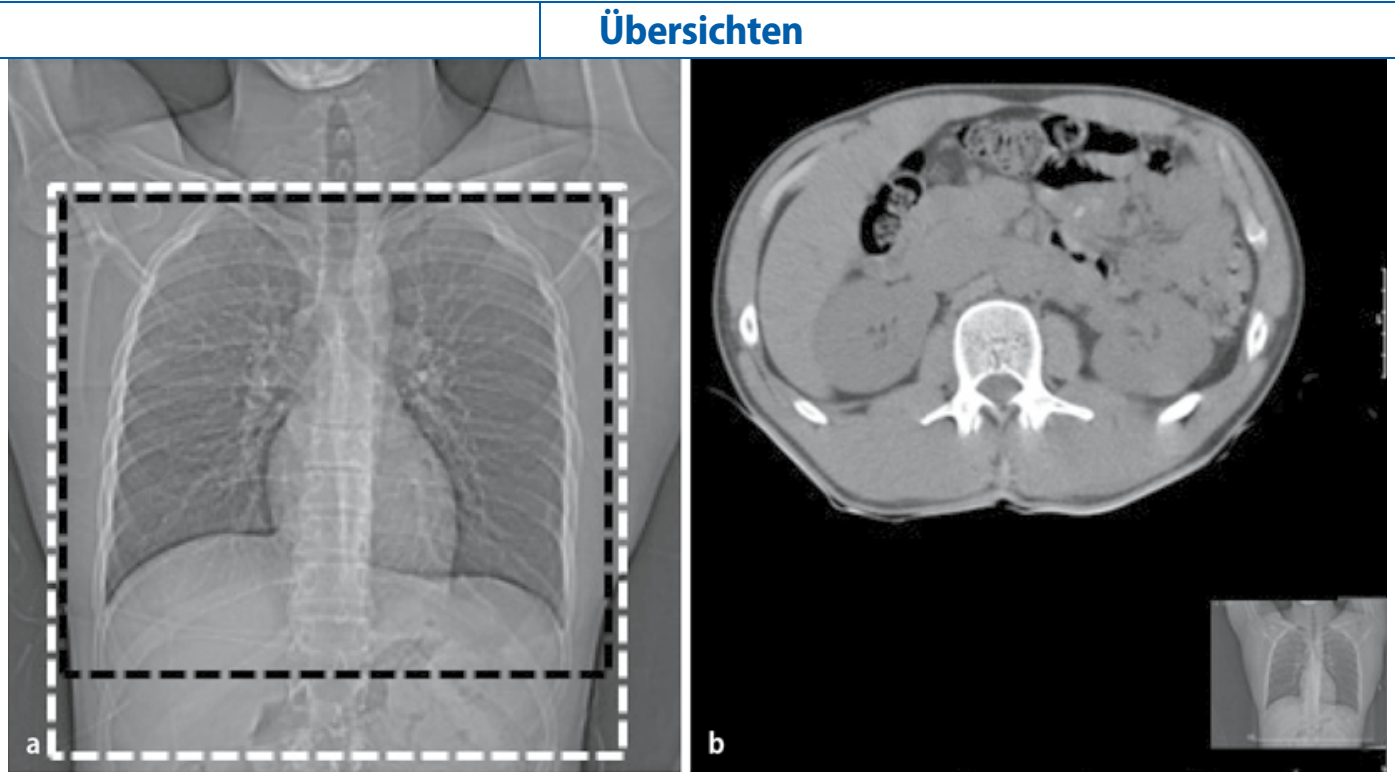

Abb. $1 \Delta$ CT-Planungsaufnahme (a) und axiale Schicht des Thorax (b) eines 26-jährigen Patienten zum Ausschluss eines Pneumothorax und einer Pneumonie. Das kaudale Ende der Scanregion ist zu großzügig definiert (weißes Rechteck in der CT-Planungsaufnahme), das kraniale Ende korrekt gewählt. Durch eine sparsamere Definition des kaudalen Endes der Scanregion (schwarzes Rechteck in der CT-Planungsaufnahme) hätte eine Strahlendosis von ca. $52 \mathrm{mGy} \times \mathrm{cm}$ (geschätzte effektive Dosis $0,83 \mathrm{mSv}$ ) eingespart werden können

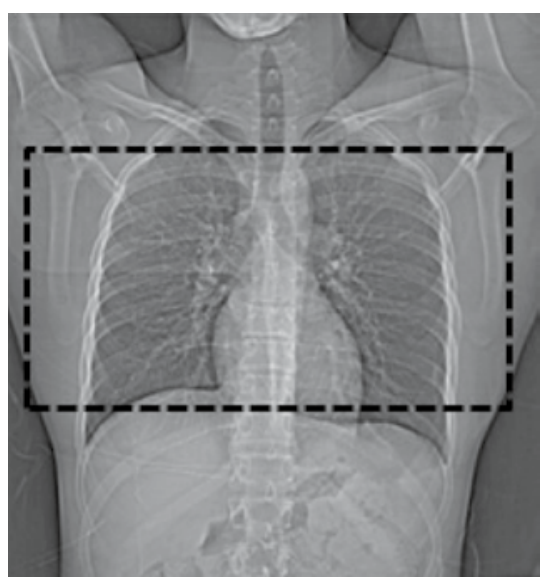

Abb. $2 \Delta$ Eingeschränktes Scanfeld (schwarzes Rechteck) für die Lungenemboliediagnostik unter Nichtberücksichtigung der apikalen und basalen Lungenabschnitte

Bei CT-Verlaufsuntersuchungen (Z. B. Tumornachsorge) sollte darauf geachtet werden, dass das Scanfeld entsprechend der Fragestellung maximal reduziert wird [7].

\section{Minimierung der Untersuchungsphasen}

Eine erhebliche Reduktion der CT-Strahlendosis wird durch die Vermeidung unnötiger Untersuchungsphasen erreicht. Mehrphasenuntersuchungsprotokolle sind nur bei sehr wenigen Fragestellungen indiziert und sollten auf keinen Fall routinemäßig angewendet werden. Eine kürzlich vorgestellte Studie wies nach, dass bei 500 Patienten, die eine CT-Untersuchung des Abdomens und Beckens erhalten hatten, in $52 \%$ der Untersuchungen mindestens eine zusätzliche CT-Phase durchgeführt wurde, die nicht indiziert war [8], am häufigsten betraf dies eine zusätzliche spätvenöse Untersuchungsphase. Bis heute besteht noch keine Standardisierung der Anzahl der CT-Untersuchungsserien bzgl. der klinischen Fragestellung. Eine zusätzliche native Aufnahme zur kontrastverstärkten CT des Thorax oder Abdomens liefert selten Zusatzinformationen.

Für folgende Fragestellungen ist eine zusätzliche native CT-Untersuchung des Thorax oder Abdomens indiziert:

- Wandhämatom der Aorta,

- Nebennierenraumforderung (z. B.

Nebennierenadenom),

- Unterscheidung zwischen Kontrastmittel (KM) und Verkalkung (z. B. bei Tumoren oder bei Erstuntersuchung nach Endoprothesenimplantation in die Aorta),

- Erkennung und Quantifizierung diffuser oder fokaler Verfettung der Leber.

Für folgende Fragestellungen ist eine Spätphase des Abdomens indiziert:
- Abklärung eines Lebertumors (z. B. Leberhämangiom, cholangiozelluläres Karzinom),

- Nebennierenraumforderung mit der Frage nach KM-Wash-out,

- Tumoren im Nierenparenchym (nephrographische Phase),

- Beurteilung des harnableitenden Systems im Sinne einer CT-Urographie,

- Verdacht auf Endoleak nach Aortenprothese,

- Verdacht auf Aortitis.

Die Splitbolustechnik führt ebenfalls zur Einsparung mindestens einer Kontrastmittelphase $[9,10]$. Dabei wird das i.v.KM in 2 oder 3 Phasen verabreicht. Damit stellt die Splitbolustechnik eine einfache Modifikation des KM-Protokolls dar, bei der 2 oder 3 separate CT-Untersuchungsphasen zu einer monophasischen CT-Untersuchung zusammengefasst werden. So wird z. B. bei der Abklärung polytraumatisierter Patienten mittels CT anstelle einer arteriellen und venösen KM-Phase eine kombinierte KM-Pphase akquiriert [9]. Während dieser Mischphase liegt eine gute Kontrastierung der Arterien, Venen und parenchymatösen Organe vor. Die Splitbolustechnik wird ebenfalls erfolgreich für CT-Untersuchungen der Nieren, bei denen die arterielle, nephrographische und exkretorische KM-Phase gleichzeitig aufgenommen wird, angewendet [10]. Je- 
doch ist sie zum Ausschluss von Nierentumoren nicht ganz unumstritten, da sie aufgrund der entstehenden Mischphase kleine Tumoren maskieren kann.

Die native CT-Untersuchung kann zudem bei Verwendung eines Dual-energyCT-Scanners dadurch vermieden werden, indem ein virtueller nichtkontrastierter Datensatz aus den kontrastverstärkten CT-Untersuchungsphasen generiert wird. Viel versprechende Ergebnisse wurden bereits zur Endoleakdetektion und zum Abklären von Nierentumoren mit Dual-energy-CT-Scannern veröffentlicht $[11,12,13]$. Analog dazu ist es mithilfe von Dual-energy-CT-Scannern auch möglich, einen virtuellen nichtkontrastierten Datensatz des Schädels nachträglich aus der kontrastverstärkten Serie zu rekonstruieren [14]. Ferda et al. [14] zeigten kürzlich, dass intrakanielle Blutungen mit sehr hoher Sensitivität anhand dieses virtuellen nichtkontrastierten Datensatzes detektiert werden können.

\section{Reduktion der Röhrenspannung und/oder des Röhrenstroms für native CT-Untersuchungen}

Wenn zusätzlich zur geplanten kontrastverstärkten CT- eine native CT-Untersuchung vom Thorax oder Abdomen vorgenommen werden soll, empfehlen wir, ein Niedrigdosisprotokoll zu verwenden, da ein verstärktes Bildrauschen für einen Großteil der Fragestellungen akzeptabel ist. Hierfür kann man entweder die Röhrenspannung, den -strom oder beide Parameter senken. Die Dosisoptimierung des nativen CT-Protokolls muss individuell abhängig von der Patientengröße und den bisher verwendeten Scanparametern erfolgen (z. B. Reduktion der Röhrenspannung von 120 auf $100 \mathrm{kV}$ bei konstanten mAs-Werten).

\section{Automatische Modulation des Röhrenstroms}

Mittels automatischer Röhrenstrommodulation erreicht man eine nahezu vergleichbare Bildqualität unabhängig vom Körperbau des Patienten. Die Software berechnet anhand des Topogramms oder der vorangegangen Rotation den individuell benötigten Röhrenstromwert für je-

Radiologe $2010 \cdot 50: 1120-1127 \quad$ DOI 10.1007/s00117-010-2053-2
○) Springer-Verlag 2010

S.T. Schindera · C. Nauer · R. Treier · P. Trueb · G. von Allmen · P. Vock · Z. Szucs-Farkas Strategien zur Reduktion der CT-Strahlendosis

Zusammenfassung

Die rasante technische Weiterentwicklung der CT hat in den letzten Jahren zu einer deutlichen Zunahme der diagnostischen Möglichkeiten geführt, mit dem Resultat, dass die CT-Untersuchungszahlen weltweit angestiegen sind. Dies hat ebenfalls Auswirkung auf die Strahlenexposition der Bevölkerung. Bis heute sind zahlreiche Publikationen erschienen, die gezeigt haben, dass eine Dosisreduktion erreicht werden kann, ohne dadurch die Bildqualität und Sensitivität der CT zu beeinträchtigen. Die Mehrzahl der Strategien zur Dosisoptimierung sind einfach anzuwenden und unabhängig von der Detektorkonfiguration des CT-Scanners. Im vorliegenden Übersichtsartikel werden die wichtigsten Methoden vorgestellt: indikationsabhängige Methoden (z. B. rechtfertigende In- dikation, Reduktion der Röhrenspannung für die CT-Angiographie, Wahl von Kollimation und Pitchfaktor, Minimierung der Untersuchungsphasen, Senkung der Röhrenspannung und des -stroms für die Nativphase), herstellerabhängige Methoden (z. B. automatische Röhrenstrommodulation, adaptive Filter zur Reduktion des Bildrauschens, iterative Bildrekonstruktion) und allgemeine Methoden (z. B. Patientenzentrierung im Isozentrum der CT-Gantry, Reduktion der Scanlänge, Anwendung von Röntgenschutzmitteln, Reduktion der Röhrenspannung und/oder des stroms für den CT-Planungsscan).

\section{Schlüsselwörter}

Computertomographie (CT) .

Strahlenexposition · Strahlendosisreduktion

\section{Strategies for reducing the $\mathrm{CT}$ radiation dose}

\section{Abstract}

The rapid technical advances in computed tomography have led to an increased number of clinical indications. Unfortunately, at the same time the radiation exposure to the population has also increased due to the increased total number of CT examinations. In the last few years various publications have demonstrated the feasibility of radiation dose reduction for $\mathrm{CT}$ examinations with no compromise in image quality and loss in interpretation accuracy. The majority of the proposed methods for dose optimization are easy to apply and are independent of the detector array configuration. This article reviews indication-dependent principles (e.g. application of reduced tube voltage for CT angiography, selection of the collimation and the pitch, re- ducing the total number of imaging series, lowering the tube voltage and tube current for non-contrast CT scans), manufacturer-dependent principles (e.g. accurate application of automatic modulation of tube current, use of adaptive image noise filter and use of iterative image reconstruction) and general principles (e.g. appropriate patient-centering in the gantry, avoiding over-ranging of the $\mathrm{CT}$ scan, lowering the tube voltage and tube current for survey CT scans) which lead to radiation dose reduction.

Keywords Computed tomography (CT) $\cdot$ Radiation exposure $\cdot$ Reduction 
de Rotation in der z-Achse und xy-Ebene. Da sich die Verfahren der automatischen Röhrenstrommodulation je nach CT-Herstellerfirma unterscheiden, verweisen wir bzgl. der technischen Grundlagen auf den Übersichtsartikel von McCollough et al. [15]. Bei schlanken Patienten kann dank automatischer Röhrenstrommodulation die Strahlendosis deutlich reduziert werden. Zur gleichen Zeit besteht aber die Gefahr, dass es bei adipösen Patienten ungewollt sowie unbeachtet zu sehr hohen Strahlendosen kommt [16]. Ferner sollte darauf geachtet werden, dass der Nutzer die Einstellungsparameter der automatischen Röhrenstrommodulation (z. B. „noise index“ bei General-Electric-[GE-]Scannern, „reference mAs" bei Siemens-Scannern) an die Bildqualität anpasst, die für die jeweilige Indikation erforderlich ist. Eine sachgerechte Anwendung der Technik ist notwendig, um eine maximale Reduktion der Strahlendosis zu erzielen und überhöhte Strahlendosen zu vermeiden. Radiologen und MTRA sollten nicht nur über den Nutzen der automatischen Röhrenstrommodulation Bescheid wissen, sondern auch über die potenziellen Gefahren.

Wenn das CT-Gerät älteren Baujahrs ist und folglich noch nicht über eine automatische Röhrenstrommodulation verfügt, ist es notwendig, den Röhrenstrom manuell an die Konstitution des Patienten anzupassen. Insbesondere sollte darauf geachtet werden, dass bei der Untersuchung von Kindern sowie schlanken Patienten, die einen deutlich geringeren Körperdurchmesser haben, der Röhrenstrom gesenkt wird, um eine unnötig hohe Strahlenexposition zu vermeiden. Der zu verwendende Röhrenstrom für CTUntersuchungen bei Kindern kann mittels einer Formel errechnet werden: $\mathrm{mAs}$ $=($ Körpergewicht +5$) \times \mathrm{f}$ mit $\mathrm{f}=1$ für Untersuchungen des Thorax, $\mathrm{f}=1,5$ für Untersuchungen des Abdomens und Beckens und $\mathrm{f}=2-5$ für Untersuchungen des Schädels [17]. Hierbei handelt es sich nur um eine sehr grobe Abschätzung der einzustellenden mAs-Werte. Je nach CT-Hersteller muss der Faktor entsprechend angepasst werden. Dies kann anhand eines Korrekturfaktors geschehen.

\section{Optimale Patienten- zentrierung in der CT-Gantry}

Vor der CT-Untersuchung wird der Patient auf dem CT-Tisch mit Hilfe eines Linienlasers zentriert und im Isozentrum der Gantry positioniert. Klinische Studien haben ergeben, dass bis zu 95\% der Patienten, die entweder eine CT des Thorax oder des Abdomens erhielten, in der vertikalen Ebene nicht präzise positioniert wurden [18]. So betrug die vertikale Abweichung vom Isozentrum im Durchschnitt 2,6 cm (Spannweite 0,6-6,4 cm [18]). Die ungenaue Patientenpositionierung resultiert in einer Zunahme der Strahlendosis in der Körperperipherie und -oberfläche sowie in einer Reduktion der Bildqualität $[18,19,20]$. Eine vertikale Abweichung von $3 \mathrm{~cm}$ führt zu einer bis zu 18\%igen $\mathrm{Zu}$ nahme der Strahlendosis in der Körperperipherie und -oberfläche bei gleichzeitiger Zunahme des Bildrauschens um 6\% [18]. Die Ursache der zunehmenden Strahlendosis und der abnehmenden Bildqualität - hervorgerufen durch ungenaue Patientenpositionierung - liegt laut Kalra et al. [20] beim Einsatz von Formfiltern (engl. „bow-tie filter“ [18]). Diese Filter schwächen die Strahlung zentral nur minimal, in der Peripherie hingegen stark. Bei einer vertikalen Abweichung des Patienten vom Isozentrum kommt es zu einem ineffizienten Einsatz des Formfilters mit einer zu hohen Strahlung in der Peripherie. Ein Großteil der modernen CT-Scanner verwendet Formfilter.

Folglich ist eine fachgerechte Instruktion der MTRA hinsichtlich einer präzisen Patientenpositionierung notwendig, um die maximal mögliche Dosisreduktion mit Formfilter dennoch zu erzielen.

\section{Reduktion der Röhrenspannung für die CT-Angiographie}

Eine niedrige Röhrenspannung (10o oder $80 \mathrm{kV}$ ) ist insbesondere für die CT-Angiographie (CTA) zur Reduktion der Strahlendosis zu empfehlen. Die Senkung des $\mathrm{kV}$-Werts führt nicht nur zu einer deutlichen Strahlenreduktion, sondern gleichzeitig auch zu einer stärkeren Kontrastierung der Gefäße aufgrund einer höheren Absorption des jodhaltigen CT-Kontrastmittels [21]. In der CTA erzielt man bei niedrigen $\mathrm{kV}$-Werten im Vergleich zu höheren die gleiche Bildqualität (KontrastRausch-Verhältnis) mit einer geringeren Strahlendosis oder eine bessere Bildqualität bei gleicher Strahlendosis [21]. Basierend auf unseren klinischen Erfahrungen und der aktuellen wissenschaftlichen Literatur empfehlen wir, routinemäßig eine Röhrenspannung von $100 \mathrm{kV}$ für die CTA der Pulmonalarterien (Ausschlussdiagnostik einer Lungenembolie) und für die CTA der thorakoabdominalen Aorta $\mathrm{zu}$ verwenden $[22,23,24,25]$. Bei der pulmonalen CTA kann eine Röhrenspannung von $80 \mathrm{kV}$ für Patienten mit einem Körpergewicht $<100 \mathrm{~kg}$ in Erwägung gezogen werden, mit der Folge, eine noch deutlichere Strahlenreduktion zu erzielen $[26,27]$. Für die kardiale CT wird ebenfalls eine Röhrenspannung von $100 \mathrm{kV}$ bei normalgewichtigen Patienten empfohlen $[28,29]$.

\section{Thorakoabdominale CT für Polytraumatisierte - Positionierung der Arme oberhalb des Schultergürtels}

Die diagnostische Abklärung polytraumatisierter Patienten wird in den meisten Kliniken mittels thorakoabdominaler CT durchgeführt. Zu dieser Patientengruppe zählen häufig junge Erwachsene sowie Kinder, die bei einer Strahlenexposition einem erhöhten Risiko ausgesetzt sind. Bei der Lagerung des Patienten auf dem CT-Tisch wird oftmals darauf verzichtet, die Arme oberhalb des Schultergürtels $\mathrm{zu}$ positionieren. Es konnte gezeigt werden, dass polytraumatisierte Patienten, die mit einer thorakoabdominalen CT unter Verwendung der automatischen Röhrenstrommodulation untersucht und bei denen die Arme seitlich des Oberkörpers gelagert wurden, eine um bis zu $45 \%$ erhöhte Strahlendosis im Vergleich zu Patienten, bei denen die Arme oberhalb des Schultergürtels positioniert wurden, erhielten [30]. Darüber hinaus hatte die Lagerung der Arme seitlich des Oberkörpers eine deutlich schlechtere Bildqualität im Oberbauch (insbesondere Leber und Milz) zur Folge.

In Einzelfällen ist eine Positionierung der Arme oberhalb des Schultergürtels wegen Frakturen oder anderen Verlet- 
zungen nicht möglich. Zudem wird in absoluten Notfallsituationen auf das zeitaufwendige Procedere der Armumlagerung verzichtet. Jedoch sollte darauf geachtet werden, wenn es aus medizinischen Gründen möglich ist, die Arme oberhalb des Schultergürtels zu lagern, da so eine deutliche Strahlenreduktion erreicht werden kann.

\section{Abschirmung der CT-Röntgen- strahlen mit Schutzmitteln}

Insbesondere bei Kindern und jungen Erwachsenen sollten die strahlenempfindlichen Organe wie z. B. die Augenlinsen, das Brustdrüsengewebe, die Schilddrüse und die Gonaden bestmöglich vor Röntgenstrahlen geschützt werden $[31,32]$. Die Anwendung von Hodenkapseln führt bei einer CT-Untersuchung des Abdomens $\mathrm{zu}$ einer um 95\% reduzierten Strahlendosis der Hoden und stellt eine einfache und kostengünstige Methode zur Dosisreduktion dar [31]. Da Hodenkapseln ausgeprägte Aufhärtungsartefakte induzieren können, sollte bei der Frage nach inguinalen Lymphknoten auf die Verwendung dieser Schutzmittel verzichtet werden. Die Anwendung von Brustschildsystemen bei Frauen während einer Thorax-CT-Untersuchung konnte sich trotz deutlicher Dosisreduktion um bis zu 50\% nicht durchsetzen, da die Abdeckung gleichzeitig zu einer deutlichen Verschlechterung der Bildqualität (Zunahme des Bildrauschens um bis zu 40\%) führt [33,34]. Wenn ein Brustschildsystem zum Einsatz kommt und gleichzeitig die automatische Röhrenstrommodulation eines GE-Scanners verwendet wird, sollte die CT-Planungsaufnahme ohne Brustabdeckung durchgeführt werden, um die maximale Dosisreduktion zu erreichen [35]. Das Auflegen des Brustschildsystems vor der CT-Planungsaufnahme hat keinen negativen Effekt auf die Dosisreduktion bei CT-Scannern der Firma Siemens [34]. Da bisher keine Daten zu CT-Scannern der Firmen Toshiba und Philips vorliegen, empfehlen wir, die CT-Planungsaufnahme ebenfalls ohne Brustschildsysteme anzufertigen.

Basierend auf unseren eigenen, nicht publizierten Daten raten wir, insbesondere bei Kindern oder jüngeren Erwachsenen, generell den Bereich, der nicht zum

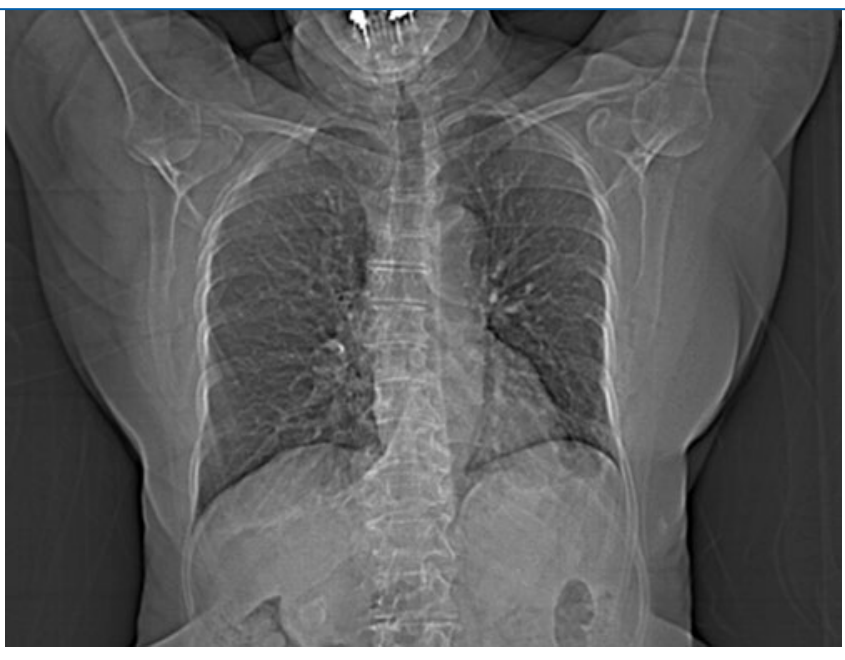

Untersuchungsfeld gehört (z. B. Mamma, Darm, Becken), mit einer Röntgenschürze abzudecken. Dadurch kann die CTStreustrahlung deutlich reduziert werden. Wann immer die Halsweichteile nicht direkt im Nutzstrahlenbündel liegen, bieten sich als kostengünstige Lösung wieder verwendbare Schilddrüsenabdeckungen mit o,5 mm Bleiäquivalent an, wie sie z. B. auch vom Personal in der interventionellen Radiologie getragen werden. So kann bei der CT des Schädels die Schilddrüsendosis durch den Einsatz einer Schilddrüsenabdeckung deutlich reduziert werden [36]. Diese Schutzmaßnahmen sollten im Sinne eines Kompromisses zwischen Aufwand und Nutzen und unter Mitberücksichtigung hygienischer Anforderungen ergriffen werden.

\section{Wahl von Kollimation und Pitchfaktor}

Bei der Kollimation sollten die Untersuchungsparameter darauf abgestimmt werden, ob ein echter isotroper Datensatz (Schichtdicke $<1$, o mm) für die Diagnostik notwendig ist oder ob auch ein nahezu isotroper Datensatz (Schichtdicke: 1,O1,5 mm) ausreicht. Unsere klinischen Erfahrungen haben gezeigt, dass ein nahezu isotroper Datensatz mit einer Schichtdicke von 1,5 mm für die Diagnostik bei Routineuntersuchungen des Thorax und Abdomens ausreicht. Für die Befundung sollten in erster Linie die dicken Schichten (Schichtdicke: 3,0-5,0 mm) genutzt werden, da diese ein deutlich besseres Signal-Rausch-Verhältnis bieten. Die dicken Schichten werden aus den primär akqui- rierten dünnen Schichten rekonstruiert. Die dünnen Schichten sollten ausschließlich zur Erzeugung dicker, multiplanarer Reformationen und zur Beurteilung unklarer Befunde in den dicken Schichten (z. B. Lungenrundherd, Partialvolumen) verwendet werden.

Der Pitchfaktor sollte wenn immer möglich größer als 1 sein, da es sonst zu überlappenden Schichten kommt, was zu einer unnötig hohen Strahlendosis führt. Ein Pitchfaktor größer als 2 sollte ebenfalls vermieden werden, da dieser zu einer lückenhaften Akquisition führt. Pitchfaktoren zwischen 1 und 2 (idealerweise 1,2 bis 1,5 ) stellen einen sinnvollen Kompromiss zwischen der Sensitivität bei der Detektion gering kontrastierter Läsionen, Bildqualität und Strahlendosis dar [37, 38].

Bei Siemens- oder Philips-Scannern hat die Wahl des Pitchfaktors (Verhältnis Tischvorschub zu Kollimation) nahezu keinen Einfluss auf die Strahlendosis, da diese beiden Herstellerfirmen mit dem pitchadaptierten Röhrenstromzeitprodukt (effektive mAs) arbeiten. Dies bedeutet, dass beim Anstieg des Pitchfaktors automatisch das Röhrenstromzeitprodukt angehoben und die Scanzeit reduziert werden, bei nahezu konstanter Dosis. Andere Hersteller, wie z. B. General Electric und Toshiba, arbeiten mit der konventionellen Definition des Röhrenstromzeitprodukts. Folglich hat die Wahl des Pitchfaktors bei diesen beiden Herstellerfirmen großen Einfluss auf die Strahlendosis. 
Tab. 1 Checkliste für MTRA und Radiologen mit den wichtigsten Methoden zur Reduktion der CT-Strahlendosis

\begin{tabular}{|c|c|}
\hline $\begin{array}{l}\text { MTRA sollten vor jeder } \\
\text { CT-Untersuchung } \\
\text { folgende Punkte } \\
\text { berücksichtigen }\end{array}$ & $\begin{array}{l}\text { Kontrolle der Scanlänge hinsichtlich möglicher Einschränkungen } \\
\text { Optimale Patientenzentrierung } \\
\text { Senken der Röhrenspannung und/oder des Röhrenstroms für die } \\
\text { CT-Planungsaufnahme } \\
\text { Positionieren der Oberarme bei einer CT-Untersuchung des Thorax und } \\
\text { Oberbauchs, wenn immer möglich, oberhalb des Oberkörpers } \\
\text { Einsatz von Schutzmitteln zur Abschirmung von CT-Röntgenstrahlen }\end{array}$ \\
\hline $\begin{array}{l}\text { Radiologen sollten bei } \\
\text { der Festlegung der CT- } \\
\text { Protokolle folgende } \\
\text { Punkte beachten }\end{array}$ & $\begin{array}{l}\text { Prüfen der rechtfertigenden Indikation } \\
\text { Mögliche Reduktion der Untersuchungsphasen } \\
\text { Senken der Röhrenspannung und/oder des Röhrenstroms für native } \\
\text { CT-Untersuchungen } \\
\text { Senken der Röhrenspannung auf } 100 \text { oder } 80 \mathrm{kV} \text { für die CT-Angiographie } \\
\text { Vermeiden einer sehr dünnen Kollimation ( }<1,0 \mathrm{~mm}) \\
\text { Vermeiden eines Pitchfaktors }<1 \text { (nur bei General Electric und Toshiba) }\end{array}$ \\
\hline
\end{tabular}

\section{Adaptive Filter und neuartige Bildrekonstruktionsverfahren zur Reduktion des Bildrauschens}

In den letzten Jahren wurden sowohl von den CT-Hersteller- als auch Softwarefirmen adaptive Filter entwickelt, die in einem Nachbearbeitungsverfahren das Bildrauschen signifikant reduzieren können. Da diese Filter das Bildrauschen bis $\mathrm{zu}$ 50\% reduzieren können, ist es möglich, Niedrigdosis-CT-Untersuchungen des Thorax und Abdomens in der Bildqualität eines Normaldosisprotokolls durchzuführen $[39,40,41,42,43]$. Diese Nachbearbeitungsfilter können direkt in das PACS (Picture Archiving and Communication System) integriert werden.

Zudem bieten die meisten CT-Herstellerfirmen in ihren aktuellen CT-Scannern völlig neuartige Bildrekonstruktionsverfahren an, die anstatt mit der herkömmlichen Standardmethode, der „filtered back projection", mit einem iterativen Bildrekonstruktionsverfahren arbeiten (Adaptive Statistical Iterative Reconstruction [ASIR] bei GE und Iterative Reconstruction in Image Space [IRIS] bei Siemens). Diese neuartigen Verfahren zeigen eine Dosisreduktion bis zu $65 \%$ bei gleich bleibender Bildqualität $[44,45,46]$.

\section{Reduktion der Röhrenspannung und/oder des Röhrenstroms für die CT-Planungsaufnahme (Scan Projection Radiograph)}

Die von der CT-Planungsaufnahme (von verschiedenen Herstellerfirmen unterschiedlich genannt, z. B. Pilot View, Sca- nogram, Scout etc.) herrührende Dosis wird oftmals vernachlässigt. Demgegenüber konnte gezeigt werden, dass je nach Gerät und Technik die applizierte Dosis bei einer CT-Planungsaufnahme des Thorax viel größer als bei einer konventionellen Thoraxaufnahme ist [47]. Ebenso können bei Planungsaufnahmen für die Schädel-CT höhere Dosen als bei einer konventionellen Aufnahme resultieren [48]. Da für die Planung der CT-Untersuchung eine reduzierte Bildqualität ausreichend ist, verwenden wir in unserem Institut eine Röhrenspannung von $80 \mathrm{kV}$ in Verbindung mit einem geringen Röhrenstrom (10-50 mA, • Abb. 3). Zudem führt die Positionierung der Röntgenröhre unterhalb anstatt oberhalb des CTTischs während der CT-Planungsaufnahme zu einer Dosisreduktion $[47,48]$.

\section{Fazit für die Praxis}

Bei der gegenwärtigen Diskussion über das durch eine CT-Untersuchung hervorgerufene Krebsrisiko sollte nicht vergessen werden, dass der Nutzen einer klinisch sorgfältig indizierten CT-Untersuchung das potenzielle Risiko wesentlich überwiegt. Es ist die Pflicht des Radiologen und der MTRA, die CT verantwortungsvoll, d. $h$. im Sinne des ALARA-Prinzips, einzusetzen. Die in diesem Artikel vorgestellten Methoden zur CT-Strahlenreduktion sollen helfen, schnell und einfach das gewünschte Ziel der Dosisoptimierung zu erreichen. In $\bullet$ Tab. 1 sind die wichtigsten Strategien zur Dosisreduktion in Form einer Checkliste zusam- mengefasst, die bei der täglichen Arbeit mit der CT eingesetzt werden kann.

\section{Korrespondenzadresse \\ PD Dr. S.T. Schindera

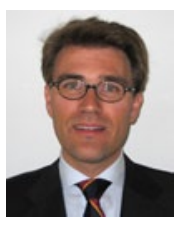 \\ Institut für Diagnostische, \\ Interventionelle und \\ Pädiatrische Radiologie, \\ Universitätsspital Bern, \\ Inselspital, \\ Freiburgstraße, CH-3010 Bern, \\ Schweiz \\ sschindera@aol.com}

Interessenkonflikt. Der korrespondierende Autor weist auf folgende Beziehung hin: Dr. Schindera erhielt Drittmittel im Rahmen eines Kooperationsvertrags von Siemens Healthcare Sector.

\section{Literatur (Auswahl)}

1. Brenner DJ, Hall EJ (2007) Computed tomography - an increasing source of radiation exposure. $\mathrm{N}$ Engl J Med 357:2277-2284

2. Berrington Gonzalez A de, Mahesh M, Kim KP et al (2009) Projected cancer risks from computed tomographic scans performed in the United States in 2007. Arch Intern Med 169:2071-2077

3. Strahlenschutzkommission (2006) Orientierungshilfe für radiologische und nuklearmedizinische Untersuchungen. ISBN 3-87344-130-6 2006

4. American College of Radiology Appropriateness Criteria: http://www.acr.org/secondarymainmenucategories/quality_safety/app_criteria.aspx

5. Kalra MK, Maher MM, Toth TL et al (2004) Radiation from „extra" images acquired with abdominal and/ or pelvic CT: effect of automatic tube current modulation. Radiology 232:409-414

6. Prokop M (2008) Radiation dose in computed tomography. Risks and challenges. Radiologe 48:229-242

7. Campbell J, Kalra MK, Rizzo S et al (2005) Scanning beyond anatomic limits of the thorax in chest $\mathrm{CT}$ : findings, radiation dose, and automatic tube current modulation. AJR Am J Roentgenol 185:15251530

8. Guite K, Hinshaw JL, Ronallo F, Lee F (2009) RSNA Scientific Meeting

9. Loupatatzis C, Schindera S, Gralla J et al (2008) Whole-body computed tomography for multiple traumas using a triphasic injection protocol. Eur Radiol 18:1206-1214

10. Kekelidze M, Dwarkasing RS, Dijkshoorn ML et al (2010) Kidney and urinary tract imaging: triple-bolus multidetector $\mathrm{CT}$ urography as a one-stop shop - protocol design, opacification, and image quality analysis. Radiology 255:508-516

11. Stolzmann P, Frauenfelder T, Pfammatter T et al (2008) Endoleaks after endovascular abdominal aortic aneurysm repair: detection with dual-energy dual-source CT. Radiology 249:682-691

12. Graser A, Johnson TR, Hecht EM et al (2009) Dual-energy $C T$ in patients suspected of having renal masses: can virtual nonenhanced images replace true nonenhanced images? Radiology 252:433440 
13. Chandarana H, Godoy MC, Vlahos I et al (2008) Abdominal aorta: evaluation with dual-source dual-energy multidetector CT after endovascular repair of aneurysms - initial observations. Radiology 249:692-700

14. Ferda J, Novak M, Mirka H et al (2009) The assessment of intracranial bleeding with virtual unenhanced imaging by means of dual-energy CT angiography. Eur Radiol 19:2518-2522

15. McCollough CH, Bruesewitz MR, Kofler JM Jr (2006) CT dose reduction and dose management tools: overview of available options. Radiographics 26:503-512

16. Schindera ST, Nelson RC, Toth TL et al (2008) Effect of patient size on radiation dose for abdominal MDCT with automatic tube current modulation: phantom study. AJR Am J Roentgenol 190:W100W105

17. Stover B, Rogalla P (2008) CT examinations in children. Radiologe 48:243-248

18. Li J, Udayasankar UK, Toth TL et al (2007) Automatic patient centering for MDCT: effect on radiation dose. AJR Am J Roentgenol 188:547-552

19. Li J, Udayasankar UK, Toth TL et al (2008) Application of automatic vertical positioning software to reduce radiation exposure in multidetector row computed tomography of the chest. Invest Radiol 43:447-452

20. Kalra MK, Maher MM, Kamath RS et al (2004) Sixteen-detector row CT of abdomen and pelvis: study for optimization of z-axis modulation technique performed in 153 patients. Radiology 233:241249

21. Schindera ST, Nelson RC, Yoshizumi T et al (2009) Effect of automatic tube current modulation on radiation dose and image quality for low tube voltage multidetector row $\mathrm{CT}$ angiography: phantom study. Acad Radiol 16:997-1002

22. Heyer CM, Mohr PS, Lemburg SP et al (2007) Image quality and radiation exposure at pulmonary CT angiography with $100-$ or $120-k V p$ protocol: prospective randomized study. Radiology 245:577-583

23. Schueller-Weidekamm C, Schaefer-Prokop CM, Weber M et al (2006) CT angiography of pulmonary arteries to detect pulmonary embolism: improvement of vascular enhancement with low kilovoltage settings. Radiology 241:899-907

24. Wintersperger B, Jakobs T, Herzog P et al (2005) Aorto-iliac multidetector-row CT angiography with low kV settings: improved vessel enhancement and simultaneous reduction of radiation dose. Eur Radiol 15:334-341

25. Kalva SP, Sahani DV, Hahn PF, Saini S (2006) Using the K-edge to improve contrast conspicuity and to lower radiation dose with a 16-MDCT: a phantom and human study. J Comput Assist Tomogr 30:391-397

26. Szucs-Farkas Z, Kurmann L, Strautz T et al (2008) Patient exposure and image quality of low-dose pulmonary computed tomography angiography: comparison of 100- and $80-\mathrm{kVp}$ protocols. Invest Radiol 43:871-876

27. Szucs-Farkas Z, Strautz T, Patak MA et al (2009) Is body weight the most appropriate criterion to select patients eligible for low-dose pulmonary CT angiography? Analysis of objective and subjective image quality at $80 \mathrm{kVp}$ in 100 patients. Eur Radiol 19:1914-1922

28. Bischoff B, Hein F, Meyer T et al (2009) Impact of a reduced tube voltage on $\mathrm{CT}$ angiography and radiation dose: results of the PROTECTION I study. JACC Cardiovasc Imaging 2:940-946
29. Leschka S, Stolzmann P, Schmid FT et al (2008) Low kilovoltage cardiac dual-source CT: attenuation, noise, and radiation dose. Eur Radiol 18:18091817

30. Brink M, Lange F de, Oostveen LJ et al (2008) Arm raising at exposure-controlled multidetector trauma CT of thoracoabdominal region: higher image quality, lower radiation dose. Radiology 249:661670

Das vollständige Literaturverzeichnis ..

... finden Sie in der html-Version dieses Beitrags im Online-Archiv auf der Zeitschriftenhomepage www.DerRadiologe.de

\section{DGHO und ESMO 2010: DKG-Internetportal bietet Orientierung für Ärzte}

Die Kongressberichte des DKG-Internetportals geben Ärzten einen zeitnahen und kompakten Überblick über neue onkologische Entwicklungen in Forschung und Praxis. So steht die Bewertung aktueller Studien diesmal im Mittelpunkt der Berichterstattung von der Gemeinsamen Jahrestagung der Deutschen, Österreichischen und Schweizerischen Gesellschaften für Hämatologie und Onkologie (DGHO) in Berlin. Im Fokus: hämatologische Tumoren, Mammakarzinom und gastrointestinale Tumoren. Außerdem online: Ein interdisziplinäres Expertengespräch zum aktuellen Stand der Therapie des Multiplen Myeloms.

Vom Kongress der European Society for Medical Oncology (ESMO) in Mailand bietet das DKG-Portal Orientierung in den aktuellen Diskussionen zu Mammakarzinom, Bronchialkarzinom, gastrointestinalen Tumoren und zur Uroonkologie.

Informationen zur aktuellen Kongressberichterstattung unter www.krebsgesellschaft.de/ arzt 